\title{
Image Acquisition Dimensionality
}

National Cancer Institute

\section{Source}

National Cancer Institute. Image Acquisition Dimensionality. NCI Thesaurus. Code

C116126.

The number of dimensions that the acquired image(s) are presented in. 